\title{
Seabed rough surface scattering described by 2- way coupled mode approach
}

\author{
D. P. Knobles ${ }^{\mathrm{a}}$ and J. D. Sagers ${ }^{\mathrm{a}}$ \\ ${ }^{a}$ Applied Research Laboratories \\ The University of Texas at Austin \\ PO Box 8029 Austin, Texas 78713-8029
}

\begin{abstract}
A 2-way integral equation coupled mode (IECM) approach is used to examine the physics of scattering from a rough seabed. Roughness realizations for the seabed are generated from a wavenumber power spectrum whose parameterization includes a roll-off wavenumber $K_{L}$ and a root mean square (rms) roughness height $h$. For the waveguide, source depth, frequency, and seabed type examined, large losses can occur due to scattering of low angle modes into the modal continuum. This occurs for small $K_{L}$ and large $h$ values. However, for large $K_{L}$ and small $h$ energy lost from the trapped modes to the continuum and backscattering can be less than the energy saved by a transfer of energy within the trapped spectrum to the lower order modes with smaller attenuation. The study suggests a critically important relationship between the modal interference distances and $1 / K_{L}$ (a correlation distance) in the physics of multimodal scattering from a rough seabed in shallow water.
\end{abstract}

Keywords: Coupled modes, rough surface scattering, seabed acoustics

PACS: 43.20.Fn

\section{INTRODUCTION}

In a previous work the integral coupled mode equations for the modal amplitudes, formulated in Cartesian coordinates, were separated into forward and backward going components. ${ }^{1}$ It was argued that a meaningful separation could be accomplished by splitting the Green's function operator into parts that satisfied the asymptotic boundary conditions. In addition to the ability to examine the individual forward and backward modal amplitudes, the splitting of the coupled mode equations laid the foundation for the construction of an energy conserving 1-way equation with the introduction of a non-local mode coupling operator. ${ }^{1}$

The purpose of this study is to apply the split IECM approach to account for the physics of acoustic propagation in a shallow water waveguide with a seabed that has a rough water-sediment interface. A roughness realization of the bottom bathymetry is generated from a 1-D roughness wavenumber spectrum that includes such parameters as a roll-off wavenumber $K_{L}$ and rms roughness height $h$. For some combinations of $K_{L}$ and $h$ the roughness causes the scattering of low angle modes into the continuum, which acts as a propagation loss mechanism due to the rapid attenuation of continuum modes with range. Another loss mechanism associated with the roughness is the transfer of acoustic energy from the forward propagating field to the backward propagating field. Examples will be provided that show these loss effects. However, 
depending on the propagation frequency, the water sound speed profile, the average properties of the seabed, and the source depth, certain combinations of $K_{L}$ and $h$ can cause the long-range depth integrated transmission loss to be less than that predicted by the adiabatic case (no mode coupling or scattering). The results of this study appear to contradict what is commonly accepted as a general rule that a rough seabed surface always results in greater propagation loss.

\section{FORMULATION}

Following the idea in Ref. 1 the 2-D coupled mode equations of dimension $2 N$ for the forward and backward modal amplitudes $\left(R^{\infty}(r)=\left[R_{1}^{\infty}, R_{2}^{\infty}, \ldots, R_{N-1}^{\infty}, R_{N}^{\infty}\right]\right.$ and $\left.R^{0}(r)=\left[R_{N+1}^{0}, R_{N+2}^{0}, \ldots, R_{N+N-1}^{0}, R_{2 N}^{0}\right]\right)$ in cylindrical coordinates are

$$
\begin{aligned}
& R_{n}^{\infty}(r)=\Gamma_{n}^{\infty}(r)+\int_{0}^{r} r^{\prime} G_{n}^{\infty}\left(r, r^{\prime}\right) \sum_{m=1}^{N} C_{n m}\left(r^{\prime}\right) R_{m}^{\infty}\left(r^{\prime}\right) d r^{\prime}+\int_{0}^{r} r^{\prime} G_{n}^{\infty}\left(r, r^{\prime}\right) \sum_{m=N+1}^{2 N} C_{n m}\left(r^{\prime}\right) R_{m}^{0}\left(r^{\prime}\right) d r^{\prime} \\
& R_{n}^{0}(r)=\Gamma_{n}^{0}(r)+\int_{r}^{\infty} r^{\prime} G_{n}^{0}\left(r, r^{\prime}\right) \sum_{m=1}^{N} C_{n m}\left(r^{\prime}\right) R_{m}^{\infty}\left(r^{\prime}\right) d r^{\prime}+\int_{r}^{\infty} r^{\prime} G_{n}^{0}\left(r, r^{\prime}\right) \sum_{m=N+1}^{2 N} C_{n m}\left(r^{\prime}\right) R_{m}^{0}\left(r^{\prime}\right) d r^{\prime}
\end{aligned}
$$

where

$$
\begin{aligned}
& \Gamma_{n}^{\infty}(r)=\int_{0}^{r} r^{\prime} G_{n}^{\infty}\left(r, r^{\prime}\right) \rho_{n}\left(r^{\prime}\right) d r^{\prime} \\
& \Gamma_{n}^{0}(r)=\int_{r}^{\infty} r^{\prime} G_{n}^{0}\left(r, r^{\prime}\right) \rho_{n}\left(r^{\prime}\right) d r^{\prime}
\end{aligned}
$$

and with $\rho_{n}$ the modal source function for the $\mathrm{n} t h$ mode and $C_{n m}$ the mode coupling matrix operator. One should exercise caution in interpreting $R_{n}^{0}(r)$ as entirely the backscattered modal amplitudes since the boundary condition for $r=0$ produces $R_{n}^{0}(r) \propto \mathrm{J}_{0}$, the zero-order Bessel function. The mathematical form of the coupling matrix operator that leads to energy conservation is not shown here, but may be found in Refs. 2-3. $N$ is the total number of modes and includes both trapped and leaky modes. $G_{n}^{0}$ and $G_{n}^{\infty}$ are the Green's functions that satisfy

$$
\left[\frac{\partial^{2} r}{\partial r^{2}}+\frac{1}{r} \frac{\partial}{\partial r}+k_{n}^{2}(r)\right] G_{n}\left(r, r^{\prime}\right)=-\frac{\delta\left(r-r^{\prime}\right)}{r},
$$

where $k_{n}(r)$ is the horizontal wavenumber eigenvalue for the $\mathrm{n} t h$ mode at range $r$. The solution to Eq. 2 is

$$
\begin{aligned}
& G_{n}^{\infty}\left(r, r^{\prime}\right)=y_{n}^{(0)}\left(k_{n} r\right) y_{n}^{(\infty)}\left(k_{n} r^{\prime}\right)\left(r^{\prime} W\left[y_{n}^{(0)}, y_{n}^{(\infty)}\right] r=r^{\prime}\right)^{-1} r<r^{\prime} \\
& G_{n}^{0}\left(r, r^{\prime}\right)=y_{n}^{(0)}\left(k_{n} r^{\prime}\right) y_{n}^{(\infty)}\left(k_{n} r\right)\left(r^{\prime} W\left[y_{n}^{(0)}, y_{n}^{(\infty)}\right] r=r^{\prime}\right)^{-1} r>r^{\prime}
\end{aligned}
$$


where $y_{n}^{(0)}\left(k_{n} r\right)$ and $y_{n}^{(\infty)}\left(k_{n} r\right)$ are independent solutions to the homogeneous version of Eq. 2 that satisfy the boundary conditions at $r=0$ and $r=\infty$, respectively. The Wronskian is

$$
W\left[y_{n}^{(0)}, y_{n}^{(\infty)}\right]_{r=r^{\prime}}=W\left[J_{0}\left(k_{n} r\right), H_{0}\left(k_{n} r\right)\right]_{r=r^{\prime}}=\frac{2 i}{\pi r^{\prime}} \text {. }
$$

The solution to Eq. 1 allows one to compute the transmission loss (TL) in the rangedepth plane as

$$
\begin{aligned}
& T L^{\infty}(r, z)=20 \log _{10}\left|\sum_{n=1}^{N} R_{n}^{\infty}(r) \Phi_{n}(z)\right| \\
& T L^{0}(r, z)=20 \log _{10}\left|\sum_{n=N+1}^{2 N} R_{n}^{0}(r) \Phi_{n-N}(z)\right| \\
& T L(r, z)=20 \log _{10}\left|\sum_{n=1}^{N}\left(R_{n}^{\infty}(r)+R_{n+N}^{0}(r)\right) \Phi_{n}(r, z)\right|
\end{aligned}
$$

where $\Phi_{n}(r, z)$ is the depth dependent mode function for the $\mathrm{n} t h$ mode at range $r$. The coupled equations in Eq. 1 are solved for $R_{n}^{\infty}(r)$ and $R_{n}^{0}(r)$ with an approach first developed by Udagawa and Kim for problems in nuclear structure ${ }^{4-5}$ and then for applications in underwater acoustics. ${ }^{6-7}$

\section{APPLICATION TO SCATTERING FROM ROUGH SEABED}

Numerical computations from the IECM approach are presented to illustrate scattering mechanisms resulting from a rough seabed. The background waveguide is $50 \mathrm{~m}$ deep with a stratified water sound speed profile (SSP) over an infinite half-space. The SSP has a thermocline depth at about $20 \mathrm{~m}$ and was one measured during the Office of Naval Research (ONR) sponsored Shallow Water 2006 experiment off the coast of New Jersey. The geoacoustic parameter values describing the bottom halfspace are $1645 \mathrm{~m} / \mathrm{s}, 2.0 \mathrm{~g} / \mathrm{cm}^{3}$, and $0.4 \mathrm{~dB} / \lambda$ for the sediment compressional sound speed, the density, and the attenuation, respectively. These geoacoustic parameters are consistent with a hard water-saturated sand. The maximum (computational) range in the waveguide is $10 \mathrm{~km}$, and the source is located at $r=0 \mathrm{~m}$ and depth $10 \mathrm{~m}$.

The roughness wavenumber power spectrum employed to generate a 1-D rough seabed realization is

$$
P(k)=\frac{K_{L} h^{2}}{\pi\left(K_{L}^{2}+k^{2}\right)^{\gamma / 2}}
$$

where $h, K_{L}, \gamma$, and $k$ are the rms roughness height, the roll-off wavenumber given by the reciprocal of the roughness correlation length, the scattering exponent, and the spatial wavenumber, respectively. A previous analysis ${ }^{8}$ of backscatter acoustic data with a 2-D spectrum in a sand environment gives an approximate value of $\gamma=2.0$ for the 1-D spectrum which we adopt in this study. A thorough study of scattering in the $K_{L} h$ plane parameter space as a function of frequency is beyond the scope of the present manuscript. Instead, we examined a small number of points in the $K_{L} h$ plane 
at an acoustic propagation frequency of $200 \mathrm{~Hz}$. As a guide for selecting $K_{L}$ and $h$ values, Thorsos and Perkins ${ }^{9}$ characterized seabed roughness as typical for $K_{L}=0.00251 / \mathrm{m}$ and $h=0.316 \mathrm{~m}$ and rough-rough for $K_{L}=0.11 / \mathrm{m}$ and $h=0.178 \mathrm{~m}$.

For the current preliminary study scattering effects were computed at (1) $K_{L}=0.00251 / \mathrm{m}$ with $\mathrm{h}$ varying from 0.5 to $2.0 \mathrm{~m}$ and (2) $\mathrm{h}=0.5 \mathrm{~m}$ with $K_{L}$ varying from $0.00251 / \mathrm{m}$ to $0.11 / \mathrm{m}$. For the numerical computations the roughness power spectrum is employed to generate a rough seabed for $2.5<r<7.5 \mathrm{~km}$, and in the regions $0.0<r<2.5 \mathrm{~km}$ and $7.5<r<10.0 \mathrm{~km}$ the waveguide is range-invariant.

Figure 1 shows $T L(r, z), T L^{\infty}(r, z)$, and $T L^{0}(r, z)$ for $K_{L}=0.00251 / \mathrm{m}$ and $h=2.0 \mathrm{~m}$. For this waveguide and frequency there are 5 trapped modes and 8 continuum (leaky) modes for a total of 13 modes. The bathymetry has been superimposed on the TL images. One observes that $T L^{\infty}(r, z)$ and $T L(r, z)$ are nearly identical because the backscattered field in $T L^{0}(r, z)$ is small. An examination of $T L(r, z)$ from $0<r<2.5 \mathrm{~km}$ shows the expected rapid decay of the continuum modes with increasing range. From $2.5<r<7.5 \mathrm{~km}$ there is strong scattering into the modal continuum, as evident by significant amounts of high angle acoustic intensity present in the bottom half-space. Some of this sound intensity in the half-space can scatter back into the trapped spectrum via higher order scattering, but most is lost to attenuation in the continuum. The depth-integrated transmission loss (DITL) at $10 \mathrm{~km}$ is $58 \mathrm{~dB}$. The DITL at $10 \mathrm{~km}$ for the adiabatic case (no mode coupling or scattering) is $50.83 \mathrm{~dB}$. If we define $\Delta$ as the 2-way DITL minus the adiabatic DITL, then $\Delta=$ $7.17 \mathrm{~dB}$. This is the additional DITL that occurs as a result of mode coupling from the rough seabed surface. 


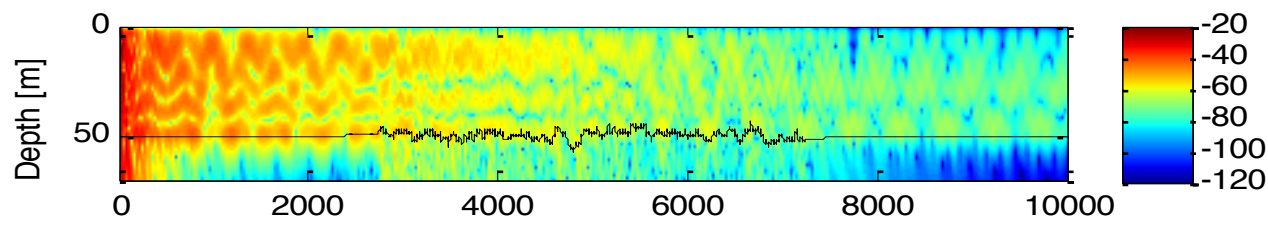

(a)

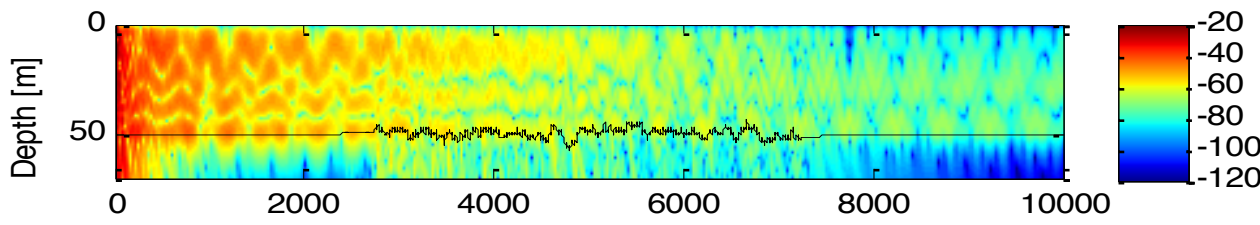

(b)

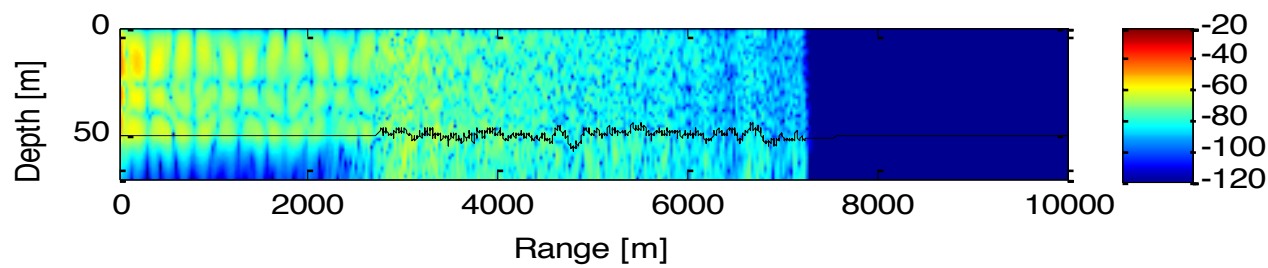

(c)

FIGURE 1. (a) $T L(r, z)$, (b) $T L^{\infty}(r, z)$, and (c) $T L^{0}(r, z)$.

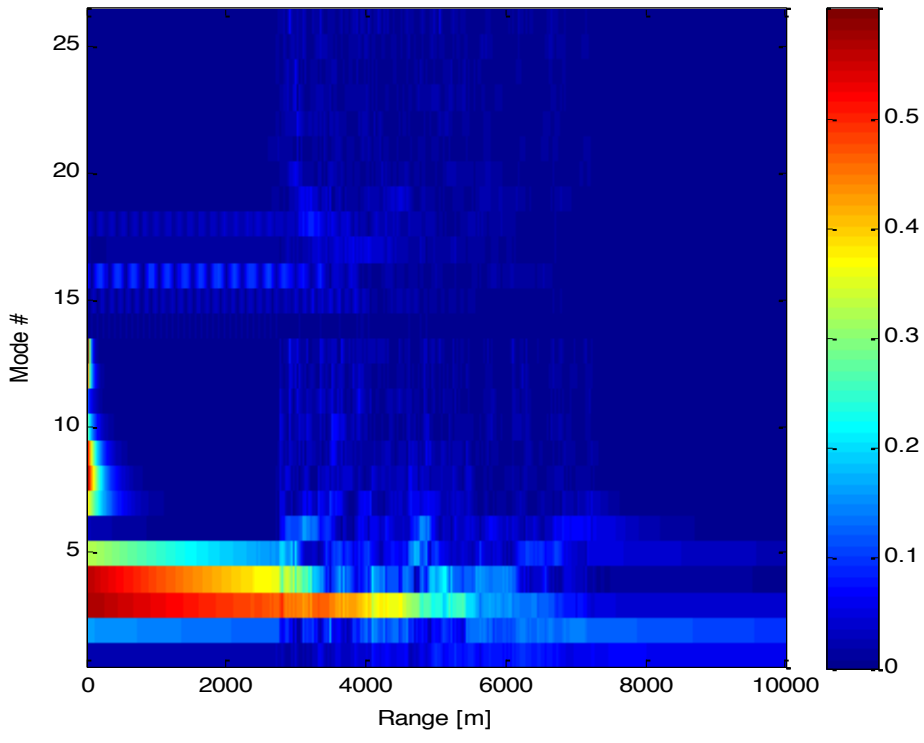

FIGURE 2. Modal amplitudes versus range.

For the purpose of giving additional physical insight into Fig. 1, Fig. 2 shows $\left|R_{n}(r)\right|$. The energy in the trapped modes is clearly shown to scatter into both the continuum modes and into the backward propagating modes (modes 14-26 on the vertical axis). 
Figure 3 shows $\Delta(h)$ for $K_{L}=0.00251 / \mathrm{m}$ and Fig. 4 shows $\Delta\left(K_{L}\right)$ for $h=0.51 / \mathrm{m}$. For $K_{L}=0.00251 / \mathrm{m}, \Delta$ increases with increasing $h$. This is not unexpected, since the mode coupling increases with increasing $h$, and it is the mode coupling that induces scattering into the continuum and the backward propagating modes. The details of this increase in $\Delta$ with $h$ will of course depend on the water column and sediment characteristics, the propagation frequency, and the source depth. In Fig. $4 \Delta$ becomes negative at about $K_{L}=0.061 / \mathrm{m}$ and continues to decrease for increasing $K_{L}$. An examination of the modal amplitudes showed that more energy was being scattered into mode 3 than was being scattered into the continuum and into the backscattering part of the modal spectrum. The distance between interference peaks for modes 3 and 4 is $\mathrm{ID}_{34}=2 \pi /\left|\operatorname{Re}\left(\mathrm{k}_{3}\right)-\operatorname{Re}\left(\mathrm{k}_{4}\right)\right| \approx 70 \mathrm{~m}$. For small $K_{L}, \mathrm{ID}_{34}$ is small as compared to $1 / K_{L}$ which can be viewed as a spatial correlation distance. As $K_{L}$ increases this spatial correlation decreases and becomes on the order of $\mathrm{ID}_{34}$ and other modal combinations within the trapped spectrum. More generally when the modal interference distance is small compared to $1 / K_{L}$ the large number of modal interference peaks over a correlation length allow for a significant transfer of energy between these modes to occur. Such scattering will be favored over pairs of modes that have a larger ID value. This appears to be the physical mechanism at work in Figs. 1 to 2 . However, as $K_{L}$ increases the correlation distance decreases and the preference for scattering into the continuum from the trapped spectrum diminishes. This appears to be the explanation for the functional form of $\Delta\left(K_{L}\right)$ in Fig. 4.

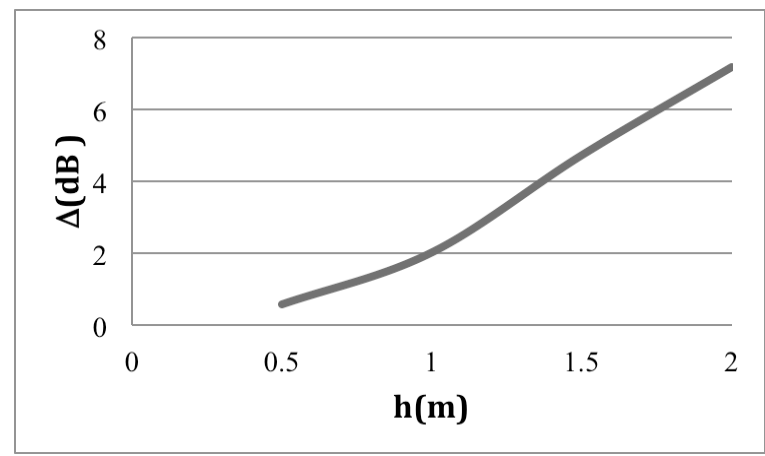

FIGURE 3. $\Delta(\mathrm{h})$ for $K_{L}=0.00251 / \mathrm{m}$.

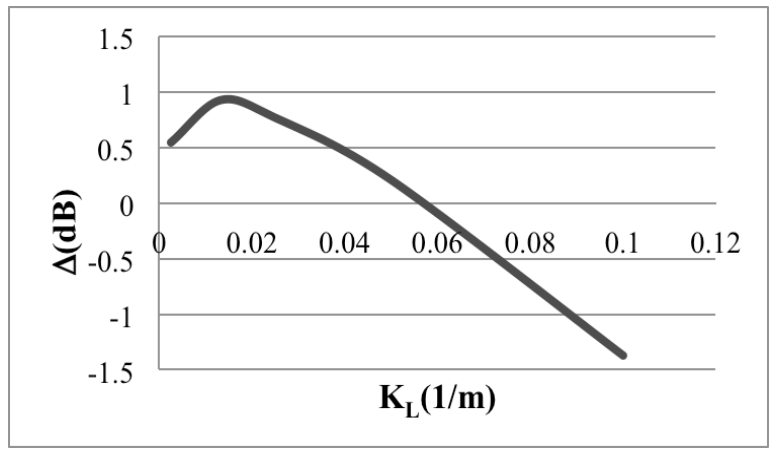

FIGURE 4. $\Delta\left(K_{L}\right)$ for $\mathrm{h}=0.5 \mathrm{~m}$. 
To establish the uncertainty in Fig. 4, the coupled mode equations were solved for 20 roughness realizations for selected $K_{L}$ values. The result was that $\sigma=0.76$ at $K_{L}=0.1$ $1 / \mathrm{m}$ and $\sigma=0.48$ at $K_{L}=0.0181 / \mathrm{m}$. The important point is that the magnitude of $\sigma$ at both values of $K_{L}$ exceeded the standard deviation and thus the mode coupling effect that allows $\Delta$ to become negative is independent of the roughness realization at $K_{L}=$ $0.1 \mathrm{1} / \mathrm{m}$.

\section{CONCLUSIONS}

A 2-way integral equation coupled mode approach provided a means to study the physical mechanisms of scattering from a rough seabed in a shallow water ocean waveguide. A 1-D roughness power spectrum whose parameterization includes a rolloff wavenumber $K_{L}$ and an rms roughness height $h$ generated a realization of bottom bathymetry. The sensitivity of the difference $(\Delta)$ of the depth integrated transmission loss and the adiabatic loss in the far-field of the waveguide to $K_{L}$ and $h$ was examined. It was found that for small $K_{L}, \Delta$ increased with $h$, whereas for a fixed value of $h, \Delta$ could become negative because energy lost to the continuum and backscattering can be less than the energy saved by a transfer of energy within the trapped spectrum to the lower order modes with smaller attenuation. This limited study of the dependence of the modal scattering on $K_{L}$ and $h$ at a single frequency revealed the complex nature of the scattering and demonstrated that the widely held belief that rough surface scattering will always increase transmission loss is not valid. The authors were surprised by this result. A more comprehensive investigation is needed on the dependence of the scattering over a wider range of $h$ and $K_{L}$ values and how the nature of the scattering varies with frequency. More specifically a better understanding is needed of the energy transfer between modes over spatial scales defined by a correlation distance in the roughness power spectrum.

\section{ACKNOWLEDGMENTS}

The U. S. Office of Naval Research supported this work. The authors have benefited from discussions with Dr. Robert Koch.

\section{REFERENCES}

1. D. P. Knobles and J. D. Sagers, "A nonlocal effective operator for coupling forward and backward propagating modes in inhomogeneous media,” J. Acoust. Soc. Am 130, 2673-2680 (2011).

2. R. A. Koch, "Coupled mode formalism in three dimensions," ARL-TL-EV-10-01 (2010).

3. Jason D. Sagers, "Predicting Acoustic Intensity Fluctuations Induced by Nonlinear Internal Waves in a Shallow Water Waveguide," Ph.D. Thesis, University of Texas at Austin, 2012.

4. T. Udagawa and B. T. Kim, "Random phase approximation calculations of nuclear response in the continuum," Phys. Rev. C 40, 2271 (1989).

5. D. P. Knobles and T. Udagawa, "Continuum random phase approximation calculations using a finite range interaction,” Nucl. Phys. A533, 189 (1991).

6. D. P. Knobles, "Solutions of coupled-mode equations with a large dimension in underwater acoustics," J. Acoust. Soc. Am. 96, 1741 (1994). 
7. D. P. Knobles, S. A. Stotts, R. A. Koch, and T. Udagawa, "Integral equation coupled mode approach applied to internal wave problems," J. Comp. Acoust. 9, 149-167 (2001).

8. K. L. Williams, D. R. Jackson, E. I. Thorsos, D. Tang, and K. B. Briggs "Acoustic backscattering experiments in a well-characterized sand sediment: Data/model comparisons using sediment fluid and Biot models," IEEE J. Ocean. Eng. 27, 376-387 (2002).

9. E. I. Thorsos and J. Perkins, "Overview of the reverberation modeling workshops," in Proceedings of the International Symposium on Underwater Reverberation and Clutter, September (2008). 
Copyright of AIP Conference Proceedings is the property of American Institute of Physics and its content may not be copied or emailed to multiple sites or posted to a listserv without the copyright holder's express written permission. However, users may print, download, or email articles for individual use. 